\title{
Evaluation of pain and catheter-related bladder discomfort relative to balloon volumes of indwelling urinary catheters: A prospective study
}

\author{
Ahmed S. Zugail ${ }^{1,2}$, Ugo Pinar ${ }^{1}$, Jacques Irani $^{1}$ \\ 'Department of Urology, Bicêtre Hospital, Le Kremlin-Bicêtre, France, 'Department of Surgery, Faculty of Medicine in Rabigh, King Abdulaziz University, Jeddah, Saudi Arabia
}

Purpose: To evaluate patients' tolerance to indwelling urinary catheters (IUCS) before and after reducing their balloon volumes. IUCs are a source of discomfort or pain.

Materials and Methods: All consecutive patients hospitalized in our department with IUCs were included during the study period with some exclusion. Each patient was his/her own control before and two hours after reduction of the balloon volume (RBV) by half using two types of assessments, a visual analog scale for pain and a catheter-related bladder discomfort (CRBD) symptom questionnaire.

Results: Forty-nine patients were included in our study that completed the assessments. The mean scores for pain before and after RBVs were 2.80 and 2.02 , respectively. The difference was significant $(p<0.05)$. The mean grades of the CRBD before and after RBVs were 1.02 and 0.75 , respectively. The difference was significant $(p<0.05)$.

Conclusions: A 50\% RBV has shown a significant amelioration in tolerating the catheter.

Keywords: Clinical protocols; Pain management; Treatment outcome; Urinary bladder; Urinary catheters

This is an Open Access article distributed under the terms of the Creative Commons Attribution Non-Commercial License (http://creativecommons.org/licenses/by-nc/4.0) which permits unrestricted non-commercial use, distribution, and reproduction in any medium, provided the original work is properly cited.

\section{INTRODUCTION}

The indwelling urinary catheter (IUC) is seen in daily medical practice for numerous etiologies. Due to its invasive nature, it is a cause of discomfort and/or pain. These symptoms are a non-negligible component of postoperative pain given that many patients are catheterized for various interventions especially urological endoscopic operations. IUC's can furthermore be indicated outside the immediate operative context. We can mention in a non-xhaustive manner the establishment of IUC's in secondary hemorrhage after endoscopic resections, in the situation of acute urinary retention awaiting management and in monitoring diuresis and effectiveness of hydration in the case of blood loss. Despite the diverse medications that oppose catheter-related bladder discomfort (CRBD), many uncertainties persist in clinical practice. All proposed effective treatments for CRBD have adverse effects and some of these effects are severe. Additionally, they may be contraindicated either permanently or in the immediate

Received: 2 September, 2018 • Accepted: 10 November, 2018

Corresponding Author: Ahmed S. Zugail

Department of Urology, Bicêtre Hospital, 78 Rue du Général Leclerc, Le Kremlin-Bicêtre 94270, France

TEL: +33-145213674, FAX: +33-145212170, E-mail: zugail2@hotmail.com

ORCID: https://orcid.org/0000-0003-3803-2115 
postoperative period. Thus, the improvement brought by this type of management is not constant and it is most often partial. The symptoms described by the patients bearing IUC's mimic those described in overactive bladder i.e., urinary frequency and urgency with or without the sensation of urge incontinence. These symptoms are probably linked in part to the stimulation of vesical muscarinic receptors by the IUC whose most voluminous intravesical part is represented by the balloon. This explains that the current management, mainly pharmacological, most often uses muscarinic receptor antagonist (MRA) that have shown their effectiveness in several studies to improve the tolerance of IUC's [1-3]. However, this approach has proven disadvantages related to the undesirable adverse effects of MRA's and also their occasional ineffectiveness owed by the presence of other mechanisms of discomfort and pain that seem to be unrelated to muscarinic receptors. This also explains the presence of many publications that propose treating these symptoms by other medications that are independent of MRA's such as paracetamol [4], tramadol [5], ketamine [6], gabapentin [7]. Consequently, we believe that all these various heterogeneous mechanisms play a role in CRBD syndrome. The simple impact of reduction of the balloon volume (RBV) of IUC's on these symptoms has never been evaluated to our knowledge. If this method proves to be effective, it would have the advantage of being simple, non-invasive and that its association with pharmacological treatment remains possible. CRBD syndrome is defined as having the urge to urinate and/or suprapubic discomfort caused by IUC's. Agarwal et al. [8] and Tauzin-Fin et al. $[9,10]$ have proposed a grading classification according to the intensity of this discomfort that can start from a simple unpleasant feeling of urgency to a permanent painful desire to urinate which could end up causing severe behavioral responses such as confusion, flailing limbs, strong vocal sounds and agitation that could lead to a traumatic attempt to remove one's own IUC. These responses may result in the increased incidence of postoperative complications, including surgical incision dehiscence, bleeding, circulatory system instability, arrhythmia, and increased severity of coronary artery disease [11]. Additionally, they can bring about exacerbated postoperative pain and prolonged hospital stay. Patients may also report other symptoms such as contractions, urethrovesical spasms, and poorly defined pain.

\section{MATERIALS AND METHODS}

This prospective, non-randomized, study was conducted after approval from the Ethics Committee of Bicêtre Hospital (approval number: KB2017-74) and written informed consent was taken from the patients. We included all consecutive patients of either gender that were hospitalized in our service with IUC's at postoperative day 1 or first day of hospitalization. The study was completed in 5 months (from September 2017 to January 2018). The exclusion criteria were patients who underwent radical prostatectomies and orthotopic bladder reconstructions because the surgeons did not want to take any chance losing the IUC's prematurely. Urinary catheterizations were done using 18-French three-way Foley catheters after applying a water-based lubricating gel to facilitate catheterization. The balloon was inflated with a variable volume of distilled water (depending on the surgeon's prescription). The IUC's were fixed to either thigh by an adhesive tape without any traction and were always left to drain freely into a urinecollecting bag. The investigator-health professional-would use a questionnaire to accumulate data from each candidate patient before and 2 hours after RBV. All questionnaires were completed between 12 and 24 hours after the insertion of IUC's. RBV is defined as reducing the IUC balloon volume by $50 \%$. To do so, $100 \%$ of the balloon's volume was deflated using a scaled syringe in milliliters without moving the IUC and then by re-inflating $50 \%$ of the obtained volume. General demographics were acquired including age, gender, height and weight. The primary objective was to compare a 10-point visual analogic scale (VAS) scores for pain before and after RBV. The secondary objective was to compare CRBD's three grades (Table 1) and questionnaire items before and after RBV. The items included symptoms of CRBD that were categorized into no (0), mild (1-3), moderate (4-6) and severe (7-10) symptoms using VAS score. The use of continuous bladder irrigation (CBI) and whether or

Table 1. Catheter-related bladder discomfort syndrome [8-10]

\begin{tabular}{ll}
\hline \multicolumn{1}{c}{ Positive diagnosis } & \multicolumn{1}{c}{ Urinary urgency and/or suprapubic discomfort } \\
\hline Grade I (mild) & Reported by the patient only on questioning. \\
Grade II (moderate) & Expressed by the patient without questioning and not accompanied by any behavioral responses. \\
Grade III (severe) & Expressed by the patient and accompanied by any behavioral responses. \\
Associated symptoms & Mandatory quantification using a pain scale. \\
& Examples: dysuria, contractions, urethrovesical spasms, poorly defined pain. \\
\hline
\end{tabular}


Table 2. Demographics of the patients

\begin{tabular}{|c|c|c|c|c|c|c|c|}
\hline Demographic & Mean & Median & Range & Interquartile range & No. of patients $(0)$ & SD & SE \\
\hline Age $(y)$ & 65.78 & 68.00 & $23-92$ & 16.50 & $49(2)$ & 14.32 & 2.05 \\
\hline Weight (kg) & 75.72 & 77.00 & $45-108$ & 17.25 & $47(4)$ & 14.75 & 2.15 \\
\hline Height $(\mathrm{cm})$ & 170.56 & 172.50 & 140-192 & 13.50 & $48(3)$ & 9.98 & 1.44 \\
\hline Balloon volume (mL) & 14.90 & 15.00 & $10-50$ & 3.00 & $48(3)$ & 6.08 & 0.88 \\
\hline Pre-RBV VAS score & 2.80 & 2.00 & $0-10$ & 5.00 & $46(3)$ & 3.05 & 0.45 \\
\hline Post-RBV VAS score & 2.02 & 1.00 & $0-7$ & 4.00 & $46(3)$ & 2.30 & 0.34 \\
\hline Pre-RBV CRBD grade & 1.02 & 1.00 & $0-3$ & 1.00 & $48(2)$ & 0.76 & 0.11 \\
\hline Post-RBV CRBD grade & 0.75 & 1.00 & $0-2$ & 1.00 & $48(2)$ & 0.76 & 0.11 \\
\hline
\end{tabular}

$\mathrm{O}$, omitted patients for insufficient data; SD, standard deviation; SE, standard error; RBV, reduction of balloon volume; VAS, visual analogic scale; CRBD, catheter-related bladder discomfort.

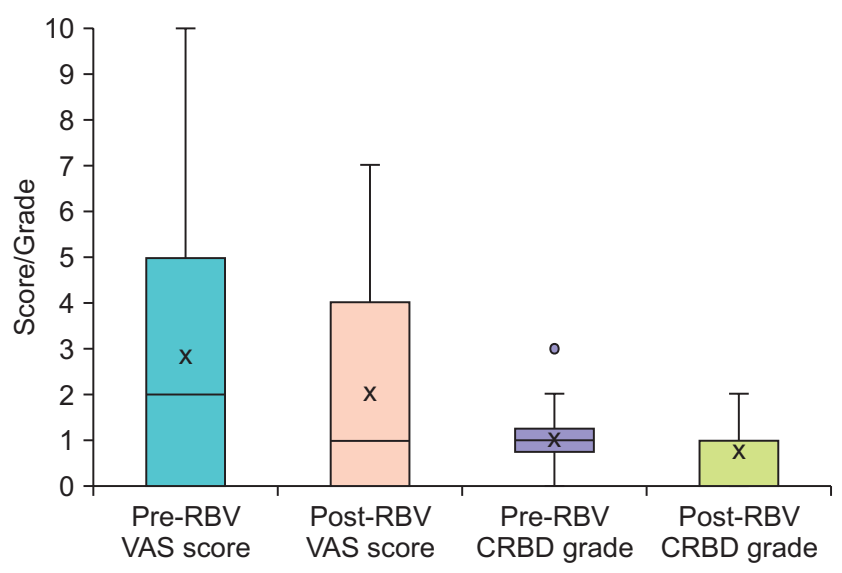

Fig. 1. Box and whisker plots displaying variation in scores and grades between VAS and CRBD groups respectively, before and after deflating the balloon. VAS, visual analogic scale; CRBD, catheter-related bladder discomfort; RBV, reduction of balloon volume.

not medications were used to alleviate any symptoms were gathered at the time of the study. Categorical variables were expressed as numbers and percentages, whereas quantitative variables were expressed by measures of central tendency, dispersion and spread. The intergroup comparisons of quantitative variables were performed using the Wilcoxon signed-rank test. A p-value of $<0.05$ was considered significant. The software StatView 5.0.1 (SAS Institute Inc, Cary, NC, USA) was used for the statistical analysis.

\section{RESULTS}

We collected data from 49 patients, 10 females and 39 males. The indications for inserting IUC's for them were as follows: After transurethral resection of bladder tumor (TURBT) 14/49 (28.6\%), after transurethral resection of the prostate (TURP) 11/49 (22.4\%), after nephrectomies either partial or complete $9 / 49$ (18.4\%), for macroscopic hematuria with clots $6 / 49$ (12.2\%), to better drain urine of transplanted patients $3 / 49$ (6.1\%), after ureteral stenting 2/49 (4.1\%), after

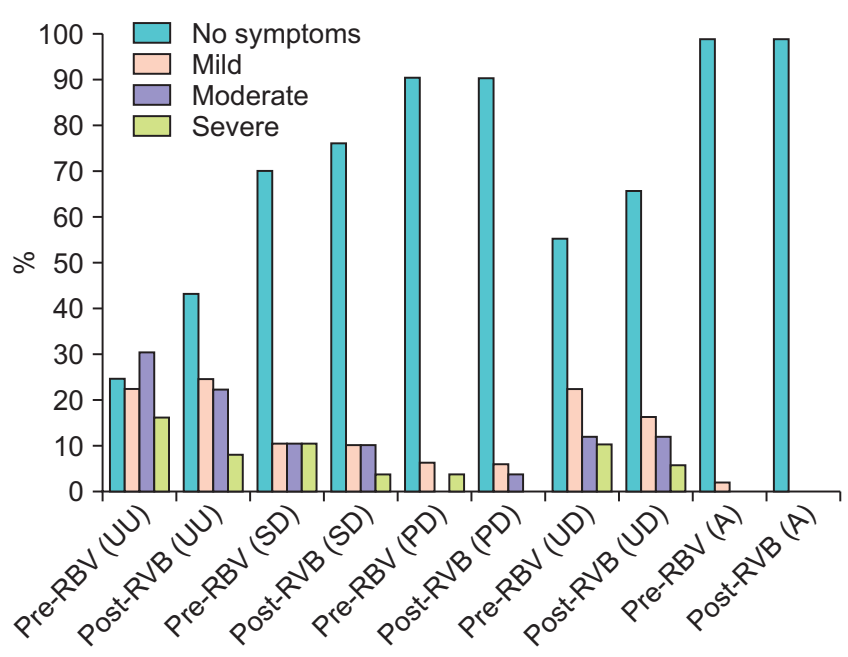

Fig. 2. A clustered column chart displaying the incidence by percentage of the severity of symptoms acquired from patients versus no symptoms before and after RBV. RBV, reduction of balloon volume; UU, urinary urgency; SD, suprapubic discomfort; $P D$, perineal discomfort; UD, urethral discomfort; $A$, agitation.

both TURBT and TURP 1/49 (2.0\%), for acute urinary retention $1 / 49(2.0 \%)$, after uterovesical fistula repair $1 / 49$ (2.0\%) and after spinal fixation with a suburethral sling $1 / 49$ (2.0\%). CBI was ongoing during the study in 24/49 (49.0\%). None of the patients received symptomatic medication during the study. No complications were reported and no IUC's were lost during or after RBV. Patients' demographics are shown in Table 2. Pre- and post-RBV VAS scores and CRBD grades are described in Table 2 and Fig. 1. Severities of symptoms acquired from patients are exhibited in Fig. 2. Comparing pre- and post-RBV symptoms in terms of VAS score, we found a significant improvement in tolerating the catheter for urinary urgency ( $\mathrm{p}$-value $=0.003$, Wilcoxon signed-rank test) but non-significant for suprapubic discomfort ( $p$-value $=0.07$, Wilcoxon signed-rank test), perineal discomfort ( $p$-value $=0.73$, Wilcoxon signed-rank test) and urethral discomfort (p-value $=0.24$, Wilcoxon signed-rank test). 
Pre- and post-RBV agitation could not be compared due to insufficient data. The incidence of CRBD in our study was $36 / 48(75.0 \%)$ reduced to $27 / 48$ (56.3\%) after RBV.

\section{DISCUSSION}

Our study has shown that a $50 \%$ reduction of the balloon volume (RBV) leads to a significant improvement in tolerating the IUC in terms of VAS scores (p-value $=0.02$ ) and CRBD grades (p-value $=0.04$ ). A limitation of our study was that the different operations could have led to immediate postoperative local physiological changes, which could also interfere with the results of our study. The potential placebo effect of the patients knowing about RBV has been taken into account but as the aim of our study was to improve the patients' comfort, it did not matter if the effect was entirely due to RBV or if a placebo effect was associated. This study comprised a restricted number of patients (49) that may have reduced the power of the study, especially for the negative findings, such as suprapubic discomfort evaluation. The incidence of CRBD is unknown but estimated to be more than $50 \%$ after urological surgery [5-7,9,12]. The urinary bladder receives cholinergic innervation by the pelvic nerves and adrenergic innervations by the hypogastric nerve. The urinary bladder exhibits heterogeneous populations of muscarinic receptors with a predominance of M2 muscarinic receptors and a minor population of M3 receptor. The M2 receptor activation causes contraction of detrusor smooth muscles [13,14]. We hypothesize that these contractions diminish with our method. A less balloon volume reduces the number of stimulated receptors leading to lessened severity of symptoms. Based on these facts, numerous treatments, such as MRA's have been implemented with different degrees of success. Side effects include, dry mouth, facial flushing, blurred vision and constipation. MRA's could be absolutely contraindicated in certain diseases, such as closed-angle glaucoma. Sedation may occur with other medications such as ketamine, gabapentin and tramadol. Our method could have a synergistic effect if used in combination of pharmacological treatment but this needs further investigation. The predisposing factors reported in the literature are male gender (longer urethra) and the diameter of IUC's, but these data need to be confirmed [15]. The placement of a suprapubic catheter significantly reduces the importance and incidence of CRBD [16,17] maybe due to the absence of a balloon or due to the maximum allowed volume of inflation of $5 \mathrm{~mL}$ in most models.

\section{CONCLUSIONS}

We have found that RBV significantly improves the tolerance of IUC's. Further controlled studies are warranted to evaluate the efficacy of the RBV protocol with higher sample sizes. We recommend using our method before administration of pharmacological treatment because it is safe, fast, not expensive, and non-invasive.

\section{CONFLICTS OF INTEREST}

The authors have nothing to disclose.

\section{ACKNOWLEDGMENTS}

We thank all participating nurses in our Urology Department at Bicêtre Hospital.

\section{REFERENCES}

1. Diokno AC, Lapides J. Oxybutynin: a new drug with analgesic and anticholinergic properties. J Urol 1972;108:307-9.

2. Yarker YE, Goa KL, Fitton A. Oxybutynin. A review of its pharmacodynamic and pharmacokinetic properties, and its therapeutic use in detrusor instability. Drugs Aging 1995;6:243-62.

3. Clmett D, Jarvis B. Tolterodine: a review of its use in the treatment of overactive bladder. Drugs Aging 2001;18:277-304.

4. Ergenoglu P, Akin S, Yalcin Cok O, Eker E, Kuzgunbay B, Turunc $T$, et al. Effect of intraoperative paracetamol on catheterrelated bladder discomfort: a prospective, randomized, doubleblind study. Curr Ther Res Clin Exp 2012;73:186-94.

5. Agarwal A, Yadav G, Gupta D, Singh PK, Singh U. Evaluation of intra-operative tramadol for prevention of catheter-related bladder discomfort: a prospective, randomized, double-blind study. Br J Anaesth 2008;101:506-10.

6. Agarwal A, Gupta D, Kumar M, Dhiraaj S, Tandon M, Singh PK. Ketamine for treatment of catheter related bladder discomfort: a prospective, randomized, placebo controlled and double blind study. Br J Anaesth 2006;96:587-9.

7. Agarwal A, Dhiraaj S, Pawar S, Kapoor R, Gupta D, Singh PK. An evaluation of the efficacy of gabapentin for prevention of catheter-related bladder discomfort: a prospective, randomized, placebo-controlled, double blind study. Anesth Analg 2007;105:1454-7.

8. Agarwal A, Raza M, Singhal V, Dhiraaj S, Kapoor R, Srivastava A, et al. The efficacy of tolterodine for prevention of catheter-related bladder discomfort: a prospective, randomized, placebo-controlled, double-blind study. Anesth Analg 
2005;101:1065-7.

9. Tauzin-Fin P, Stecken L, Sztark F. Catheter-related bladder discomfort in post-anaesthesia care unit. Ann Fr Anesth Reanim 2012;31:605-8.

10. Tauzin-Fin P, Sesay M, Svartz L, Krol-Houdek MC, Maurette P. Sublingual oxybutynin reduces postoperative pain related to indwelling bladder catheter after radical retropubic prostatectomy. Br J Anaesth 2007;99:572-5.

11. Bai Y, Wang X, Li X, Pu C, Yuan H, Tang Y, et al. Management of catheter-related bladder discomfort in patients who underwent elective surgery. J Endourol 2015;29:640-9.

12. Agarwal A, Dhiraaj S, Singhal V, Kapoor R, Tandon M. Comparison of efficacy of oxybutynin and tolterodine for prevention of catheter related bladder discomfort: a prospective, randomized, placebocontrolled, double-blind study. Br J Anaesth 2006;96:377-80.
13. Caulfield MP, Birdsall NJ. International Union of Pharmacology. XVII. Classification of muscarinic acetylcholine receptors. Pharmacol Rev 1998;50:279-90.

14. Yamanishi T, Chapple CR, Chess-Williams R. Which muscarinic receptor is important in the bladder? World J Urol 2001;19:299-306.

15. Binhas M, Motamed C, Hawajri N, Yiou R, Marty J. Predictors of catheter-related bladder discomfort in the post-anaesthesia care unit. Ann Fr Anesth Reanim 2011;30:122-5.

16. Krane LS, Bhandari M, Peabody JO, Menon M. Impact of percutaneous suprapubic tube drainage on patient discomfort after radical prostatectomy. Eur Urol 2009;56:325-30.

17. Tewari A, Rao S, Mandhani A. Catheter-less robotic radical prostatectomy using a custom-made synchronous anastomotic splint and vesical urinary diversion device: report of the initial series and perioperative outcomes. BJU Int 2008;102:1000-4. 